\title{
COLELITÍASE E CÂNCER DE VESÍCULA BILIAR
}

\section{CHOLELITHIASIS AND GALLBLADDER CARCINOMA}

\author{
Orlando Jorge Martins Torres, $\mathrm{TCBC}-\mathrm{MA}^{1}$ \\ Lia Raquel de Alcântara Caldas ${ }^{2}$ \\ Rodrigo Palácio de Azevedo ${ }^{2}$ \\ Ricardo Lima Palácio ${ }^{2}$ \\ Maria Luisa dos Santos Rodrigues ${ }^{3}$ \\ José Anselmo Cordeiro Lopes ${ }^{4}$
}

\begin{abstract}
RESUMO: Objetivo: O presente estudo tem por objetivo analisar os achados histológicos da vesícula biliar de pacientes submetidos à colecistectomia eletiva no Hospital Universitário Presidente Dutra, São Luís-MA. Método: Foram avaliados 2.008 pacientes, 359 do sexo masculino (17,9\%) e 1.649 do sexo feminino $(82,1 \%)$, com média de idade de 46,3 anos, operados no período de janeiro de 1990 a dezembro de 1998. A vesícula biliar, imediatamente após a colecitectoma era aberta e examinada macroscopicamente pelo cirurgião e em seguida enviada para exame histopatológico. Resultados: A prevalência de câncer da vesícula biliar foi de 2,3\% (46 pacientes). Trinta e três pacientes $(71,7 \%$ ) apresentavam idade superior a 60 anos. Conclusões: Os autores concluem que a colecistectomia profilática eletiva deve ser realizada em pacientes assintomáticos com colelitíase, com idade superior a 60 anos e em boas condições cirúrgicas.
\end{abstract}

Descritores: Câncer da vesícula biliar; colelitíase; colecistectomia.

\section{INTRODUÇÃO}

A investigação histológica da vesícula biliar após colecestectomia por colelitíase, é um procedimento importante devido à possibilidade do diagnóstico de câncer. $\mathrm{O}$ câncer da vesícula biliar, dito como raro, é reconhecido como a neoplasia maligna mais comum da árvore biliar e o quinto carcinoma mais frequiente do trato gastrintestinal. A doença é encontrada em $1 \%$ a $2 \%$ das peças de colecistectomia e o diagnóstico feito de forma incidental no momento da colecistectomia em aproximadamente um terço dos $\operatorname{casos}^{1,2}$.

Devido às características clínicas inespecíficas e sintomas ausentes no câncer precoce, o diagnóstico só é possível em estágio avançado, quando o prognóstico é reservado, com índice de sobrevida em cinco anos de aproximadamente 5\% na maioria das séries estudadas ${ }^{3,4}$.
A litíase biliar é fator incontestável na etiopatogênese do câncer da vesícula biliar. Diferentes estudos mostram que a maioria dos pacientes com câncer da vesícula apresenta colelitíase e sofre da doença por um período de tempo considerável antes do diagnóstico de câncer ${ }^{4-6}$.

Sabe-se atualmente que em apenas um quarto dos pacientes os tumores da vesícula biliar são ressecados com finalidade curativa e que o prognóstico do paciente tem relação direta com a precocidade do diagnóstico. A abertura sistemática da vesícula biliar ainda no transoperatório para investigação da presença de doença neoplásica permite o tratamento definitivo de alguns pacientes com neoplasia de vesícula biliar ${ }^{7,8}$.

O objetivo deste trabalho foi avaliar as características histológicas da vesícula biliar de pacientes submetidos a colecistectomias eletivas, verificando a prevalência de câncer da vesícula biliar.

1. Professor Adjunto-Doutor e Coordenador da Disciplina de Clínica Cirúrgica III — UFMA. Professor Livre-Docente - UFC.

2. Estudante de Medicina - UFMA

3. Médico Residente - UFMA

4. Doutor em Anatomia Patológica e Professor Adjunto — UFMA

Recebido em 02/05/2001.

Aceito para publicação em 15/02/2002.

Trabalho realizado na Disciplina de Clínica Cirúrgica III da Universidade Federal do Maranhão (UFMA) 


\section{MÉTODO}

No período de janeiro de 1990 a julho de 1998, 2.008 pacientes foram submetidos à colecistectomia eletiva no Serviço de Clínica Cirúrgica do Hospital Universitário Presidente Dutra em São Luís - MA. O diagnóstico pré-operatório de colelitíase foi obtido após exames clínico, laboratorial e exames de imagem. O diagnóstico de câncer da vesícula biliar não foi realizado no pré-operatório. A vesícula biliar, imediatamente após a colecistectomia, era aberta e examinada macroscopicamente pelo cirurgião no intraoperatório e em mesa separada. Em seguida era enviada para estudo anatomopatológico.

No serviço de Anatomia Patológica a vesícula biliar era preparada em cortes de $5 \mathrm{~mm}$, embebida em parafina, seccionada e corada com hematoxilina-eosina e examinada em microscópio óptico. Todas as lâminas foram reexaminadas por um mesmo patologista.

\section{RESULTADOS}

Entre os 2.008 pacientes submetidos à colecistectomia, havia 1.649 do sexo feminino $(82,1 \%)$ e 359 do sexo masculino (17,9\%), com idade variando entre 5 e 99 anos (média de 46,3 anos).

A freqüência das lesões observadas evidenciou 2,3\% de câncer da vesícula biliar (46 pacientes) e está representada na Tabela 1.

Tabela 1

Resultado do estudo anatomopatológico da vesícula biliar.

\begin{tabular}{lrr}
\hline & $N o$ & $\%$ \\
\hline Colecistite aguda & 32 & 1,6 \\
Colecistite crônica & 1.928 & 96,0 \\
Câncer & 46 & 2,3 \\
Adenoma & 2 & 0,1 \\
\hline
\end{tabular}

Dos pacientes com laudo anatomopatológico positivo para adenocarcinoma, sete eram do sexo masculino $(15,9 \%)$ e 39 do sexo feminino $(84,1 \%)$, com idade variando de 30 a 75 anos e média de 62,1 anos. Destes pacientes, 40 apresentavam idade superior a 50 anos $(86,9 \%)$ e $33(71,7 \%)$ apresentavam idade superior a 60 anos.

Quanto ao padrão histológico do adenocarcinoma observamos que 16 eram do tipo tubular moderadamente diferenciado $(34,8 \%)$, nove tubular bem diferenciado $(19,6 \%), 14$ tubular mal diferenciado $(30,4 \%)$ e sete papilífero $(15,2 \%)$.

\section{DISCUSSÃO}

O estudo histológico da vesícula biliar é de importância relevante, pois pacientes com carcinoma de vesícula biliar podem se apresentar com colecistite aguda ou crô- nica sem suspeita de malignidade. Devido às características clínicas inespecíficas e por sintomas ausentes no câncer precoce, esta neoplasia é freqüentemente diagnosticada em estágio tardio, com um prognóstico extremamente reservado e com uma taxa de sobrevida de cinco anos de aproximadamente $5 \%$ na maioria das séries. $\mathrm{O}$ resultado do seu tratamento está diretamente relacionado com o estadiamento, obtendo-se melhores resultados com tumor em estágio precoce, o qual em um terço dos casos é um achado incidental após colecistectomia por doença benigna ${ }^{9,10}$.

Dos diversos fatores etiológicos do carcinoma de vesícula biliar estudados, a presença de litíase biliar é considerada o fator de risco mais importante. Piehler e Crichlow, analisando 2.352 pacientes com carcinoma da vesícula biliar, observaram colelitíase em $73,9 \%{ }^{11}$. Nervi et al., utilizando modelos matemáticos de regressão, observaram que pacientes com litíase biliar têm risco de câncer de vesícula biliar sete vezes maior que aqueles sem cálculos ${ }^{12}$. Hart et al. observaram que a grande maioria dos pacientes com câncer da vesícula biliar apresenta colelitíase e sofre da doença por um período considerável antes do diagnóstico do câncer da vesícula biliar ${ }^{4}$. Tem sido demonstrado que a incidência do câncer da vesícula biliar é maior nos países prevalentes em colelitíase ${ }^{11,13}$.

A ligação entre litíase vesicular e carcinoma da vesícula biliar se relaciona ao trauma crônico e à inflamação da mucosa da vesícula produzida pela presença do cálculo que induz displasia epitelial, que predispõe ao carcinoma ${ }^{4,13}$.

Alguns fatores têm sido considerados como de risco para carcinoma da vesícula biliar. Jukemura et al., estudando colelitíase e o risco de carcinoma, concluíram que o fator idade foi o que mais fortemente se correlacionou com o câncer da vesícula biliar, e a incidência de $1,68 \%$ na população geral se elevou para 3,96\% nos pacientes acima de 50 anos, $4,16 \%$ para aqueles acima de 55 anos e $5,71 \%$ em pacientes acima de 70 anos $^{14}$. No presente estudo, $86,9 \%$ dos pacientes com diagnóstico de adenocarcinoma apresentavam idade superior a 50 anos e $71,7 \%$ dos pacientes apresentavam idade superior a 60 anos.

Histologicamente, a colecistite está usualmente presente em associação com o carcinoma e quando a colecistite crônica leva a calcificação da vesícula, o risco de malignidade se eleva. O risco de câncer na "vesícula em porcelana" é muito elevado (20\%) e justifica a colecistectomia profilática ${ }^{15}$.

A dimensão do cálculo estaria relacionada com a freqüência do tumor, onde o tamanho do cálculo é proporcional à sua idade e que, portanto, cálculos maiores estariam presentes a mais tempo que os menores, o que levaria a maior tempo de exposição aos fatores cancerígenos. Assim, a litíase assintomática, principalmente com cálculos grandes, pode ser perigosa em pacientes acima de 50 anos ${ }^{12,15}$.

As neoplasias benignas da vesícula biliar, como adenoma, também têm demonstrado relação direta com o carcinoma in situ e carcinoma invasivo ${ }^{14,16-18}$ Kozuka et al., estudando 1.605 peças da vesícula biliar, observaram histologicamente: a) presença de transição de adenoma para carcinoma; b) associação de todos os carcinomas in situ com componentes adenomatosos; c) freqüência considerá- 
vel de resíduos de componentes adenomatosos; d) aumento gradual de tamanho com a frequiência de alterações de malignização; e) aumento gradual da idade média dos pacientes com adenoma benigno, adenoma com alterações pré-câncer e carcinoma invasivo sucessivamente; f) predominância do sexo feminino nos pacientes com adenoma e carcinoma invasivo. Estudos têm demonstrado que estes pólipos quando maiores que $10 \mathrm{~mm}$ de diâmetro apresentam maior potencial maligno e, se diagnosticados em pacientes assintomáticos, mesmo na ausência de cálculos, a colecistectomia está recomendada ${ }^{19}$. Os pólipos precisam ser removidos se forem sésseis mesmo se menores que $10 \mathrm{~mm}$ e pediculados se maiores que $10 \mathrm{~mm}$. Também devem ser removidos se localizados junto ao parênquima hepático em pacientes acima de 40 anos ${ }^{5,18,19}$. Entre os métodos de diagnóstico, a ultra-sonografia é provavelmente o melhor. Os principais tipos de apresentação ultra-sonográfica são massas intraluminares imóveis com a mudança de decúbito, espessamento difuso ou localizado da parede vesicular, espessamento irregular da parede da vesícula, litíase biliar, obstrução biliar e massa no hilo hepático. Dois aspectos ultra-sonográficos importantes para poder se suspeitar de câncer de vesícula biliar são cálculos fixos no fundo da vesícula (que podem estar presos por crescimento tumoral) e dilatação dos ductos biliares intra-hepáticos mesmo sem massa tumoral em loja vesicular ${ }^{20,21}$.

O tratamento dos pacientes portadores de câncer de vesícula biliar depende basicamente do estadiamento onde varia de colecistectomia videolaparoscópica a ressecções hepáticas alargadas associadas à terapia complementar sempre muito discutida ${ }^{22-27}$.

Considerando a incidência de câncer da vesícula biliar em pacientes com colelitíase, particularmente aqueles com idade superior a 60 anos, a colecistectomia eletiva deve ser sempre considerada naqueles com boas condições cirúrgicas, mesmo que assintomáticos.

\begin{abstract}
Background: The aim of the present study is to analyze histological findings of the gallbladders removed during elective cholecystectomy at Hospital Universitário Presidente Dutra, São Luís, MA. Method: From January 1990 to December 1998, 2.008 patients underwent elective cholecystectomy, 359 of them males (17,9\%) and 1.649 females $(82,1 \%)$, with a mean age of 46,3 years. The gallbladder was oppened and examined by the surgeon and sent to histological examination. Results: The prevalence of gallbladder carcinoma was 2,3\% (46 patients). Thirty-three patients $(71,7 \%)$ were over 60 years old. Conclusions: Elective prophylatic cholecystectomy should be performed in asymptomatic patients over 60 years of age with cholelythiasis and good clinical conditions.
\end{abstract}

Key words: Gallbladder carcinoma; Cholelythiasis; Cholecystectomy.

\title{
REFERÊNCIAS
}

1. Arnaud JP, Casa C, Georgezc D et al. Primary carcinoma of the gallbladder - review of 143 cases. Hepatogastroenterol, 1995, 42: 811-815.

2. Chijiiwa K, Tanaka M. Carcinoma of the gallbladder: an appraisal of surgical resection. Surgery, 1994, 115: 751756.

3. Chijiiwa K, Yamaguchi K. Clinicopathologic difference between long-term postoperative survivors with advanced gallbladder carcinoma. World J Surg, 1997, 21: 98-102.

4. Hart J, Modan B, Shani M. Cholelithiasis in the etiology of gallbladder neoplasm. Lancet, 1971, 1:1151-1153.

5. Montagnini ALM, Jukemura J, Gianini PTH et al. Gallbladder histologic findings in 221 consecutive elective cholecystectomy. HPB Surgery 1995, 9: 131.

6. Sumiyoshi K, Nagai E, Nakayama F. Patholgy of carcinoma of the gallbladder. World J Surg, 1991, 15: 315321.

7. Gall FP, Kokerling F, Scheele J, Schneider C et al. Radical operations for the carcinoma of the gallbladder: Present status in Germany. World J Surg 1991, 156:328336.
8. Jones RS. Palliative operative procedures for carcinoma of the gallbladder. World J Surg, 1991, 15:348-351.

9. Arextabala X, Roa I, Burgo L. Gallbladder cancer in Chile. Cancer, 1992, 69: 60-65.

10. Zantonski W, La Vecchia C, Levi F et al. Descriptive epidemiology of gallbladder cancer in Europe. J Cancer Res Clin Oncol, 1993, 119:165-171.

11. Piehler JM, Crichlow RW. Primary carcinoma of the gallbladder. Surg Gynecol Obstet, 1978, 147:947-942.

12. Nervi F, Duarte I, Gomez G, Rodriguez G et al. Frequency of gallbladder cancer in Chile. Int J Cancer, 1988, 41:657-660.

13. Shirai Y, Yoshida K, Tsukada K. Early carcinoma of the gallblader. Eur J Surg, 1992, 158:545-548.

14. Jukemura J, Leite KRM, Machado MCC et al. Frequency of incidental gallbladder carcinoma in Brazil. ABCD. Arq Bras Cir Dig, 1997, 12:10-13.

15. Juvonen T, Niemelä $\mathrm{O}$, Mäkelä J, et al. Characteristics of symptomatic gallbladder disease in patients with either solitary or multiple cholesterol gallstones. Hepatogastroenterol, 1994, 41:263-266. 
16. Aldridge MC, Bismuth H. Gallbladder cancer: the polyp cancer sequence. Br J Surg, 1990, 77: 363-364.

17. Chijiiwa K, Tanaka M. Polypoid lesion of the gallbladder: indications of carcinoma and outcome after surgeries for malignant polypoid lesion. Int Surg, 1994, 79:106109.

18. Nakayama F. Recent progress in the diagnosis and treatment of the carcinoma of the gallbladder. Word J Surg, 1991, 15:313-314.

19. Kozuka S, Tsubone M, Yasui A et al. Relation of adenoma to carcinoma in the gallbladder. Cancer, 1982, 50:2226-2234

20. Chijiiwa K, Sumioyoshi K, Nakayama F. Impact of recent advances in hepatobiliary imaging thechniques on the preoperative diagnosis of carcinoma of the gallbladder. World J Surg, 1991, 15: 322-327.

21. Mincis R, Schmillevitch J, Mincis M. Aspectos ultra-sonográficos do câncer primário da vesícula biliar. Arq Gastroenterol, 1994, 31:03-13.

22. Busse PM, Cady B, Stone MD. Intraoperative radiation therapy for carcinoma of the gallbladder. World J Surg, 1991, 15:352-356.

23. Gagner M, Rossi RL. Radical operations for carcinoma of the gallbladder: Present status in North America. World J Surg, 1991, 15: 344-347.

24. Landford JE, Long JM et al. Laparoscopic cholecystectomy in neoplasm of the gallbladder. Surgery, 1994, 114:131-134.

25. Ogura Y, Myzumoto R, Tabata M. Radical operations for carcinoma of the gallbladder: Present status in Japan. World J Surg, 1991, 15: 337-343.
26. Yamaguchi K, Chijiiwa K, Ichimiya H et al. Gallbladder carcinoma in the era of laparoscopic cholecystectomy. Arch Surg, 1996, 131: 981-984.

27. Torres OJM, Macedo EL, Nunes PMS et al. Câncer da vesícula biliar. Rev Bras Med, 2000, 57: 602-614.

Endereço para correspondência:

Dr. Orlando Torres

Rua Ipanema, 01, Ed. Luggano, Bl I/204 — São Francisco 65076-060 - São Luís-MA

E-mail: otorres@elo.com.br 